\title{
Proceso enfermero en neonato con retraso en la recuperación quirúrgica por atresia duodenal
}

\section{Nursing process in neonate with delay in the surgical recovery for duodenal atresia \\ Processo de enfermagem do neonato com atraso na recuperação cirúrgica por atresia duodenal}

\author{
Itzel Andrea Sánchez-Juárez ${ }^{1}$ \\ (iD) https://orcid.org/0000-0003-2979-0416 \\ María Luz de Avila-Arroyo ${ }^{2 *}$ \\ (D)https://orcid.org/0000-0003-4924-1959 \\ Inés Tenahua-Quit| ${ }^{3}$ \\ (D)https://orcid.org/0000-0002-3987-8254 \\ Alejandro Torres-Reyes ${ }^{4}$ \\ (D)https://orcid.org/0000-0001-5133-4172
}

1. Licenciada en Enfermería. Benemérita Universidad Autónoma de Puebla, Facultad de Enfermería. Puebla, México.

2. Maestra en Ciencias de Enfermería. Benemérita Universidad Autónoma de Puebla, Facultad de Enfermería. Puebla, México.

3. Maestra en Ciencias de Enfermería. Benemérita Universidad Autónoma de Puebla, Facultad de Enfermería. Puebla, México.

4. Maestro en Enfermería. Benemérita Universidad Autónoma de Puebla, Facultad de Enfermería. Puebla, México.

*Autor para correspondencia: maluz_deavila@hotmail.com

Recibido: $05 / 03 / 2020$

Aceptado: 18/01/2021

\section{Resumen}

Introducción: La atresia duodenal, es una malformación congénita donde una anormalidad anatómica o funcional ocasiona la falla del tránsito intestinal normal. El tratamiento de elección es la reparación quirúrgica, que tiene como objetivo restablecer la continuidad intestinal. Los neonatos sometidos a este tipo de procedimientos tienen alta morbilidad asociada a complicaciones quirúrgicas, sepsis y dehiscencia de herida quirúrgica. Objetivo: Implementar el proceso enfermero mediante el uso de las Taxonomías NANDA, NOC, NIC y GPC para contribuir al manejo del 
neonato con retraso en la recuperación quirúrgica por atresia duodenal. Metodología: Se abordó a través de un estudio de caso en el que se implementaron las etapas del proceso enfermero, la valoración se realizó por patrones funcionales de salud de Marjory Gordon, se priorizaron los patrones alterados e identificaron etiquetas diagnósticas. Presentación del caso: Neonato de 22 días de vida extrauterina, nació de 38.5 SDG de un hospital público, postoperada de plastia intestinal, tenía una herida quirúrgica dehiscente e infectada. Presentó signos de dolor agudo y complicaciones en la motilidad gastrointestinal como distención abdominal y residuo gástrico. Los resultados auxiliares de diagnóstico mostraron trombocitopenia y aumento de la proteína C reactiva. Conclusión: La jerarquización de los patrones funcionales afectados, permitió realizar la ejecución de un plan de cuidados y atender las respuestas humanas que el neonato presentó, a través de intervenciones sustentadas en evidencia científica.

Palabras clave: Proceso de enfermería; Recién nacido; Anomalías congénitas; Atresia intestinal; Herida quirúrgica; Dolor agudo (DeCS).

\begin{abstract}
Introduction: Duodenal atresia is a congenital malformation where a functional or anatomic abnormality causes normal intestinal motility's disorders. The definitive treatment includes a surgical repair, whose main goal is to recover the intestinal continuity. Neonates treated with this kind of procedures have a high morbidity associated to surgical complications, sepsis, and dehiscence of surgical wound. Objective: Implement the nursing process by using the NANDA, NOC, NIC, and GPC taxonomies in order to contribute to the management of the newborn with delayed surgical recovery from duodenal atresia. Methodology: It was addressed through a case study in which the stages of the nursing process were implemented; the assessment was done by patterns functional health of Marjory Gordon, the altered patterns were prioritized and diagnosis labels were identified. Case presentation: 22-day old neonate extrauterine, was born from 38.5 SDG in a public hospital, post operated on intestinal plasty; the neonate had a dehiscent and infected surgical wound. The neonate showed signs of acute pain and gastrointestinal motility complications such as bloating and gastric residue. Auxiliary diagnosis results showed thrombocytopenia and increased $C$ reactive protein. Conclusion: The hierarchical organization of the affected functional patterns allowed executing a care plan and taking care of the needs of the neonate, all of that through interventions sustained on scientific evidence.
\end{abstract}

Key words: Nursing process; newborn; Congenital abnormalities; Intestinal atresia; Surgical wound; Acute pain (DeCS).

\begin{abstract}
Abstrato
Introdução: A atresia duodenal é uma malformação congênita onde uma anormalida de anatômicaou funcional causa a falha do trânsito intestinal normal. O tratamento de escolha é o reparo cirúrgico, que visa restaurar a continuidade intestinal. Os recém-nascidos submetidos a esse tipo de procedimento a presentam alta morbidade associada a complicações cirúrgicas, sepse e deiscência da ferida cirúrgica. Objetivo: Implementar o processo de enfermagem mediante o uso das Taxonomias NANDA, NOC, NIC e GPC que contribua a o manejo do recém-nascido com atraso narecuperação cirúrgica por atresia duodenal. Metodologia: Foi abordada por meio de umestudo de caso no qual o processo de enfermagemfoi aplicado, á avaliaçãofoi realizada pelos padrõesfuncionais de saúde de Marjory Gordon,
\end{abstract}


foram priorizados os padrões alterados e foram identificadas ás etiquetas de diagnóstico. Apresentação do caso: Neonato de 22 dias de vida extra-uterina, nesceu de 38.5 SDG de um hospital público, pós-operatória de plastia intestinal, tinhauma ferida cirúrgicadeiscente e infetada. Apresentousinais de dor aguda e complicaçõesnamotilidade gastrointestinal como a distensão abdominal e resíduo gástrico. Os resultados do diagnóstico auxiliar mostraram trombocitopenia e aumento da proteína $C$ reativa. Conclusão: $A$ hierarquia dos padrõesfuncionaisafetados, permitiu realizar á execução de um plano de cuidados e atender àsrespostas humanas que o neonato apresentou, através de intervençõesbaseadas em evidências científicas.

Palavras-chave: Proceso de enfermagem; Recém-nascido; Anormalidades congênitas; Atresia intestinal; Ferida cirúrgica; Dor aguda (DeCS).

\section{Introducción}

La Organización Mundial de la Salud (OMS), define a las malformaciones congénitas, como anomalías estructurales o funcionales que ocurren durante la vida intrauterina que se detectan durante el embarazo, parto o en un momento posterior al nacimiento ${ }^{(1)}$. En México, el $59.8 \%$ de las defunciones en el período neonatal, están representadas por alguna malformación congénita, dentro de las que se encuentra la atresia del intestino delgado que se presenta en alguna porción de este, es una patología poco frecuente, que en el año 2017 se presentó en 46 casos de nacidos vivos (2).

La atresia, ocasiona la falla del tránsito intestinal normal, y tiene una prevalencia de 1 por cada 5,000 a 10,000 nacidos vivos ${ }^{(3,4)}$. Diversos estudios demuestran que alrededor de un tercio de los casos se asocian con el síndrome de Down o trisomía $21^{(4-6)}$. El diagnóstico puede realizarse precozmente por medio de una ecografía prenatal, a partir de la semana 20 de gestación, donde se observará la dilatación del estómago y la porción proximal del duodeno, lo que produce el característico signo de la "doble burbuja", así como también por la presencia de polihidramnios; posterior al nacimiento, el diagnóstico definitivo se realiza mediante una radiografía simple de abdomen ${ }^{(3,5,7)}$.

Los neonatos con atresia duodenal, presentan vómito durante las primeras horas después del nacimiento, y las características de éste dependerán de la localización de la obstrucción con respecto a la ampolla de Váter; cuando se encuentra por encima, en el vómito se observará contenido gástrico, si la obstrucción se encuentra por debajo, 
habrá pérdida de bilis y jugo pancreático; otro signo clínico es la distención abdominal a causa de la acumulación de líquidos y gases en la porción próxima a la obstrucción ${ }^{(3)}$. El tratamiento de elección es la reparación quirúrgica, que tiene el objetivo de restablecer la continuidad intestinal para permitir la alimentación, mediante una duodenoduodenostomía abierta, en forma de diamante u operación de Kimura, sin embargo, los neonatos sometidos a este tipo de procedimientos, tienen alta morbilidad asociada principalmente a complicaciones quirúrgicas, sepsis y dehiscencia de herida quirúrgica ${ }^{(3,5)}$.

Con relación a lo descrito no se encontró evidencia donde se abordará el Proceso Enfermero (PE) aplicado en esta patología en específico, sin embargo, se ubicaron investigaciones en que se estudió la atresia esofágica en neonatos y los principales diagnósticos enfermeros que desatacan son alimentación ineficaz, alteración de la nutrición, riesgo de infección y patrón del sueño alterado. Así mismo, ambos casos utilizaron la valoración del dolor con la escala CRIES (Crying, requires oxygen to maintain saturation > 95\%, increased vital signs, expression, sleepenness), y señalaron la etiqueta de dolor agudo, enfocando las intervenciones a las medidas no farmacológicas ${ }^{(8,9)}$.

Lo descrito evidencia la importancia de unificar criterios de cuidado para los neonatos en el postoperatorio del tracto gastrointestinal con retraso en la recuperación quirúrgica dado que las complicaciones comprometen su estado de salud. En este sentido, el profesional de enfermería cuenta con el $\mathrm{PE}$, un método de intervención que favorece a la práctica independiente y calidad en el cuidado ${ }^{(10)}$. Por lo tanto, surge el objetivo: implementar el PE mediante el uso de las Taxonomías NANDA, NOC, NIC y GPC para contribuir al manejo del neonato con retraso en la recuperación quirúrgica por atresia duodenal.

\section{Metodología}

Se abordó a un neonato a través de un estudio de caso en el que se aplicó el PE en sus cinco etapas valoración, diagnóstico, planeación, ejecución y evaluación en la unidad de cuidados intensivos neonatales (UCIN), de un hospital de alta especialidad del estado de Puebla, México. Se basó en el marco de valoración de los patrones funcionales de salud de Marjory Gordon (11), que se complementó con la exploración física. La validación de los datos fue mediante la 
revaloración del paciente, la participación de otros profesionales de enfermería para confirmarlos y así llegar al uso de las taxonomías de la NANDA Internacional, Inc ${ }^{(12)}$ formulando los diagnósticos enfermeros.

Posteriormente se establecieron los resultados deseados que expresan el estado, conducta o percepción de la paciente con la clasificación de resultados de enfermería (NOC) ${ }^{(13)}$, a partir de ello se determinó la clasificación de intervenciones de enfermería (NIC) ${ }^{(14)}$ y recomendaciones de Guías de Práctica Clínica (GPC) ${ }^{(15-16)}$. En la obtención de los datos, se aseguró la confidencialidad de la información con el consentimiento informado que se entregó a los padres del neonato, asimismo se brindó un trato digno y respetuoso.

\section{Presentación del caso}

\section{Antecedentes}

Se trata de un neonato de sexo femenino, quien para fines de este estudio se nombra AMG. Cuando se inicia la valoración tenía 22 días de vida extrauterina, se encontraba en la incubadora en el servicio de UCIN, con los diagnósticos médicos siguientes: post-operada de plastia intestinal (duodeno-duodeno anastomosis tipo Kimura; 17 días) por atresia duodenal, dehiscencia e infección de herida quirúrgica, síndrome colestásico y de Down. De acuerdo con los antecedentes familiares no se reportan enfermedades crónico-degenerativas ni congénitas en la familia.

Nace de 38.3 semanas de gestación por test Capurro vía abdominal, producto de la tercera gesta de su madre de 35 años, con estudios de licenciatura, ocupación docente y con un adecuado control prenatal; su padre de 32 años estudió hasta secundaria y era encargado de mantenimiento; el matrimonio tenía dos hijos de 11 y 4 años. Son de religión católica y originarios del estado de Tlaxcala que residían en Puebla.

\section{Valoración por patrones funcionales de salud de Marjory Gordon}

Se realizó una valoración general (17 de febrero de 2018) y una focalizada (18 de febrero de 2018). En la primera se recopilaron datos globales de las respuestas humanas para determinar los elementos que representaban alguna alteración y a partir de ello se llevó a cabo la segunda, donde se profundizó las alteraciones consideradas como 
prioritarias al determinar el estado de salud de la recién nacida. Posteriormente se hizo un análisis de los datos objetivos y subjetivos determinando los patrones disfuncionales, que a continuación se describen.

Patrón nutricional-metabólico: los indicadores de la antropometría de AMG registraron un peso 3500 gramos, talla $54 \mathrm{~cm}$, perímetro torácico $34.5 \mathrm{~cm}$. y perímetro abdominal $35.5 \mathrm{~cm}$. Presentó las siguientes variaciones en los resultados de la glucometría capilar: a las 8 horas $48 \mathrm{mg} / \mathrm{dl}$, a las 14 horas $56 \mathrm{mg} / \mathrm{dl}$ y a las 20 horas $61 \mathrm{mg} / \mathrm{dl}$. Se mantenía con Nutrición Parenteral Total de $72.8 \mathrm{kcal} / \mathrm{kg} /$ día a infusión continua de $15 \mathrm{ml} / \mathrm{hora}$ por vía intravenosa a través Catéter Venoso Central de Inserción Periférica (PICC). Además, se inició $2 \mathrm{ml}$ de fórmula por vía enteral a través de una sonda orogástrica (SOG) calibre 5 French ( $F r$ ) para cada tres horas; sin embargo, posterior a ello presentó distensión abdominal y residuo de $2 \mathrm{ml}$ de contenido gástrico antes de la segunda toma, por lo que se le indica ayuno, retiro de esta sonda y colocación de una de mayor calibre (8 Fr) para dejarla a derivación; a partir de lo anterior se obtuvo un gasto de características biliares de $5 \mathrm{ml}$ en 6 horas.

La piel de AMG mostraba tinte ictérico, la mucosa oral estaba hidratada, tenía boca pequeña y macroglosia lo que provocaba un reflejo de succión débil, también tuvo dificultad en la regulación de temperatura corporal que osciló entre $36.7^{\circ} \mathrm{C}$ y $37.5^{\circ} \mathrm{C}$. En mesogastrio, tenía una herida quirúrgica dehiscente, ovalada, de aproximadamente $3 \mathrm{~cm}$ de longitud por uno de ancho, con formación de fibrina y bordes difusos; se observa excavación de $1 \mathrm{~cm}$ ubicada de las 9 a las 12 de acuerdo con las manecillas del reloj, y presencia de exudado en moderada cantidad, fétido, color verdoso; la piel perilesional de la herida era eritematosa, y se encontraba cubierta con un apósito de polihexametileno biguanida (PHMB) y micropore, que sirvió para valorar el gasto de la misma. Presentó edema en miembro pélvico izquierdo aparentemente por la presencia del PICC, que al momento de la valoración tenía 15 días desde su instalación, la curación de éste se había realizado el día previo a la valoración.

Patrón eliminación: el gasto urinario que presentó fue de $4 \mathrm{ml} / \mathrm{kg} / \mathrm{hora}$, recolectado de nueve mediciones, con orina de color amarillo oscuro, tuvo dos evacuaciones con heces de características semipastosas color verde; mostraba distención y red venosa visible en abdomen, a la auscultación el peristaltismo intestinal estaba disminuido y había 
flatulencias constantes antes de las evacuaciones. Tenía sudoración frecuente posterior a los períodos de irritabilidad y llanto prolongado.

Patrón actividad- ejercicio: las constantes vitales que reportó al momento de la valoración fueron: Frecuencia cardiaca 143 lpm, respiratoria $68 \mathrm{rpm}$, temperatura $37.5^{\circ} \mathrm{C}$, saturación parcial de oxígeno $\left(\mathrm{SpO}_{2}\right) 97 \%$ y llenado capilar distal de 3 segundos. Se encontraba en fase I de ventilación con casco cefálico y humidificador con fracción inspirada de oxígeno $\left(\mathrm{FiO}_{2}\right)$ al 50\%. AMG se mostraba despierta y activa con movimientos constantes, irritable a la manipulación hasta llegar al llanto.

Patrón sueño-descanso: AMG no logra conciliar largos períodos de sueño, estos duran aproximadamente una hora.

Patrón cognitivo-perceptual: de acuerdo con la Escala de Glasgow modificada para lactantes, se identificó el estado de conciencia alerta (14/15); presenta signos de dolor a la palpación y manipulación en zona abdominal con un nivel 6 según la escala CRIES, al identificarse llanto con tono agudo inconsolable, requerimiento de $\mathrm{FiO}_{2}>30 \%$ de oxígeno, expresión facial con muecas, despertar a intervalos frecuentes.

La valoración fue continua y, cuatro días después de la primera, se identificó el reinició de la alimentación VO por SOG, posterior a la administración de tres tomas separadas en lapsos de tres horas, presentó vómito de características biliares por lo que se dejó en ayuno nuevamente. En cuanto a la valoración de la herida quirúrgica, mostraba un tamaño de $3 \mathrm{~cm}$ de longitud por un cm de ancho, con presencia de tejido granular, se encontró socavamiento de $1 \mathrm{~cm}$ de las 9 a las 12 de acuerdo con las manecillas del reloj, exudado verdoso no fétido escaso y eritema cutáneo circundante en disminución; se utilizó nuevamente un apósito de PHMB el cual se cubrió con cinta adhesiva microporosa. Resultados auxiliares diagnósticos: la citometría hemática evidencia trombocitopenia (6.0 $10 \wedge 3 / \mathrm{l})$ y leucocitosis $(4.010 \wedge 3 / \mu \mathrm{l})$, los valores de proteína $\mathrm{C}$ reactiva $(\mathrm{PCR})$ se encontraban elevados $(94.7 \mathrm{mg} / \mathrm{l})$. El hemocultivo periférico realizado el día 19 de febrero, reveló el crecimiento de bacterias Gram (+) y probable contaminación por Staphylococcus aureus, datos que sugieren el diagnóstico de sepsis neonatal (16). 
Después identificar los patrones alterados mediante la valoración se estableció un juicio clínico a través del razonamiento crítico formulando los diagnósticos enfermeros de acuerdo con la condición del neonato ${ }^{(12)}$. Con base a esto se seleccionaron las intervenciones de enfermería jerarquizándolas por medio de las necesidades humanas de Maslow y Kalish con la finalidad de lograr resultados esperados para evitar, reducir o corregir las respuestas alteradas en el neonato mediante la preparación e implementación de las intervenciones para posteriormente evaluar las etapas del PE ${ }^{(17)}$ (tabla 1).

Tabla 1. Plan de cuidados de enfermería para el neonato AMG.

\begin{tabular}{|c|c|c|}
\hline agnóstico enfermero (NANDA) & $\begin{array}{l}\text { Resultado } \\
\text { esperado } \\
\text { (NOC) }^{(13)}\end{array}$ & Intervenciones \\
\hline $\begin{array}{l}\text { Dolor agudo relacionado con } \\
\text { agentes biológicos (infección) como } \\
\text { lo demuestra conducta expresiva } \\
\text { (inquietud, irritabilidad, llanto), y } \\
\text { evidencia mediante pruebas } \\
\text { estandarizadas del dolor: escala } \\
\text { CRIES nivel 6: Ilanto con tono agudo } \\
\text { inconsolable, requerimiento de FiO } 2 \\
>30 \% \text { de oxígeno, expresión facial } \\
\text { con muecas, despertar a intervalos } \\
\text { frecuentes. }\end{array}$ & $\begin{array}{l}\text { Nivel del dolor. } \\
\text { Indicadores: } \\
\text { - Duración de los } \\
\text { episodios de } \\
\text { dolor } \\
\text { - Inquietud } \\
\text { - Irritabilidad } \\
\text { - Muecas de dolor } \\
\text { - Lágrimas }\end{array}$ & $\begin{array}{l}\text { Manejo del dolor: agudo } \\
\text { (14) } \\
\text { - Realizar una valoración } \\
\text { exhaustiva del dolor que } \\
\text { incluya la localización, } \\
\text { aparición, duración, } \\
\text { frecuencia e intensidad, así } \\
\text { como factores que lo alivian } \\
\text { y agudizan. } \\
\text { - Monitorizar el dolor } \\
\text { utilizando una herramienta } \\
\text { de medición válida y fiable } \\
\text { apropiada para la edad. }\end{array}$ \\
\hline
\end{tabular}

GPC SS-745-15 (15)

- Controlar los factores ambientales que puedan influir en la respuesta del neonato a las molestias.

- Fomentar períodos de sueño adecuados que faciliten el alivio del dolor.

- Realizar la evaluación del dolor antes y después de procedimientos clínicos dolorosos.

- Se sugiere la evaluación del dolor con la escala CRIES, cada dos horas para continuar o retirar el tratamiento instituido.

\section{Ejecución y Evaluación}

Se monitorizó el dolor mediante la escala CRIES cada dos horas. Para el control del dolor, se emplearon medidas farmacológicas, como la administración de paracetamol IV; y no farmacológicas, tales como evitar el ruido excesivo, cubrir la incubadora de la luz ambiental y el uso de la succión no nutritiva, principalmente durante la curación de la herida quirúrgica y punciones para monitorizar la glucosa capilar. Además, se respetó el ciclo de sueño/vigilia y se programaron las intervenciones con el fin de evitar estímulos repetidos. La evaluación mostró disminución en el llanto, se pudo lograr un puntaje de 5 (dolor moderado) en la escala CRIES. 
Continuación tabla $1 . .$.

\begin{tabular}{|c|c|c|c|}
\hline $\begin{array}{l}\text { Motilidad gastrointestinal } \\
\text { disfuncional relacionado con } \\
\text { cambios en los hábitos alimentarios } \\
\text { y estresores como lo demuestra } \\
\text { abdomen distendido, aumento del } \\
\text { residuo gástrico, residuo gástrico de } \\
\text { color bilioso, vómitos. } \\
\text { Condiciones asociadas: intolerancia } \\
\text { alimentaria y alimentación enteral. }\end{array}$ & $\begin{array}{l}\text { Función } \\
\text { gastrointestinal. } \\
\text { Indicadores: } \\
\text { - Tolerancia a la } \\
\text { alimentación } \\
\text { - Cantidad de } \\
\text { residuos en el } \\
\text { contenido dóstrico aspirado } \\
\text { - Color } \\
\text { contenido del } \\
\text { gástrico aspirado } \\
\text { - Distención } \\
\text { abdominal } \\
\text { - Vómitos }\end{array}$ & $\begin{array}{l}\text { Cuidados de la sonda } \\
\text { gastrointestinal (14) } \\
\text { - Observar la correcta } \\
\text { colocación de la sonda y } \\
\text { fijar la sonda a la parte } \\
\text { corporal correspondiente. } \\
\text { - Vigilar periódicamente el } \\
\text { estatus hidroelectrolítico. } \\
\text { - Monitorizar la cantidad, } \\
\text { color y consistencia del } \\
\text { contenido gástrico. } \\
\text { - Proporcionar cuidados de la } \\
\text { nariz y boca } 3 \text { a } 4 \text { veces al } \\
\text { día o cuando sea necesario } \\
\text { y cuidados a la piel de } \\
\text { alrededor. } \\
\text { Alimentación enteral por } \\
\text { sonda (14) } \\
\text { - Antes de cada alimentación } \\
\text { intermitente, comprobar si } \\
\text { hay residuos. } \\
\text { - Observar si hay presencia } \\
\text { de sonidos intestinales cada } \\
\text { 4-8 horas. } \\
\text { - Controlar la ingesta/ } \\
\text { excreción de líquidos. } \\
\text { nesar diariamente al } \\
\text { neonato. }\end{array}$ & $\begin{array}{l}\text { Se preparó el material para la } \\
\text { recolocación de la SOG, se utilizó un } \\
\text { calibre } 8 \text { Fr y posterior a la colocación, } \\
\text { se verificó que AMG no presentara } \\
\text { signos de asfixia. Se fijó la SOG en las } \\
\text { mejillas y dejó a derivación con una } \\
\text { bolsa recolectora. Se registraron las } \\
\text { características del gasto de la SOG. Se } \\
\text { realizó el aseo de la cavidad oral, de } \\
\text { manera gentil cada cuatro horas. Al } \\
\text { finalizar el turno se llevó a cabo el } \\
\text { control de líquidos, en el que se } \\
\text { obtuvo un balance positivo (+153 ml). }\end{array}$ \\
\hline
\end{tabular}


a una temperatura neutra

$\left(37^{\circ} \mathrm{C}\right)$, para propiciar una

- correcta actividad enzimática, funcionamiento y metabolismo celular.

- Ejercer una presión de lavado que garantice el arrastre de detritus, bacterias y material de curaciones previas, sin lesionar el tejido sano.

- Utilizar apósitos antimicrobianos que contribuyan a reducir el dolor y olor cuando se sospeche la progresión hacia una infección o interrupción de la cicatrización.

Severidad de la Control de infecciones (14)
infección: recién nacido. - Mantener técnicas de Indicadores: aislamiento apropiadas y precauciones universales. - Limitar el número de

- Inestabilidad de la temperatura

- Aumento de

leucocitos de

visitas y solicitar que se laven las manos al entrar y salir de la habitación.

- Garantizar

una

- Supuración fétida

- Drenaje purulento

- Irritabilidad manipulación aséptica de todas las vías intravenosas.

Regulación de temperatura (14)

- Comprobar la temperatura del neonato al menos cada 2 horas y vigilarla hasta que se estabilice.

- Observar color y temperatura de la piel.

- Ajustar la temperatura ambiental a las necesidades del neonato.

GPC SS-283-12 (16)
Se llevaron a cabo todas las precauciones por contacto que el neonato requería tales como estar en un cuarto aislado, realizar la higiene de manos durante los cinco momentos. Se vigiló la temperatura axilar cada dos horas, presentando alzas en la curva térmica, por lo que, como medida no farmacológica, se abrió la campana de la incubadora hasta que disminuyera la temperatura del neonato. Se administraron vancomicina $46 \mathrm{mg}$ cada 8 horas, meropenem $62 \mathrm{mg}$ cada 12 horas, amikacina $65 \mathrm{mg}$ cada 12 horas y fluconazol $21 \mathrm{mg}$ cada 12 horas IV

- Apoyo nutricio parenteral.

Fuente: NANDA ${ }^{(12)}$, NOC ${ }^{(13)}$, NIC ${ }^{(14)}$, GPC SS-745-15 ${ }^{(15)}$; elaboración propia. 


\section{Discusión}

El PE garantiza la sistematización del cuidado para cubrir las necesidades derivadas de las respuestas humanas. Teniendo en cuenta los diagnósticos enfermeros obtenidos de este estudio se evidenció el uso de la escala CRIES, al igual que Lara y Montero ${ }^{(8,9)}$, quienes a partir de ello determinaron la etiqueta diagnóstica de dolor agudo. Este puede ser manifestado a causa de que los neonatos con atresia fueron sometidos a procedimientos quirúrgicos que condicionan la presencia del dolor, la escala identifica parámetros fisiológicos y de comportamiento ante los constantes estímulos dolorosos que los neonatos experimentan y no se pueden obtener verbalmente, por lo que el uso de la escala permitió valorarlo, así mismo atender a la recomendación de la GPC SS-745-15 (15).

Por otra parte, en este estudio se encontró el diagnóstico motilidad gastrointestinal disfuncional, dato que no concuerda con otros autores quienes establecieron la alimentación ineficaz y alteración de la nutrición ${ }^{(8,9)}$, sin embargo, dichos hallazgos podrían relacionarse ya que ambos atienden las necesidades del tracto gastrointestinal que al estar alterado impide la ingestión, digestión y absorción de nutrientes, y las intervenciones se enfocan a mantener el estado nutricional mediante la alimentación enteral y parenteral para favorecer a la recuperación.

\section{Conclusiones}

En este estudio se implementó el PE, estableciendo como diagnósticos el dolor agudo, motilidad gastrointestinal disfuncional y retraso en la recuperación quirúrgica. Posteriormente a la ejecución del plan de cuidados se evidenció que los resultados esperados presentaron un cambio de sustancial a moderadamente comprometido. Se sugiere la utilización de la escala CRIES para valorar el dolor en el neonato. Realizar intervenciones sustentadas en evidencia científica a través del PE, otorga al personal de enfermería el uso de un lenguaje estandarizado para llevar a cabo el cuidado al neonato con retraso en la recuperación quirúrgica por atresia duodenal, así como, una atención individualizada y oportuna para mejorar el bienestar.

\section{Conflicto de intereses}

No existe conflicto de intereses entre los autores. 


\section{Financiamiento}

Financiamiento propio de los autores.

\section{Referencias bibliográficas}

1. Organización Mundial de la Salud (OMS). Nota descriptiva de anomalías congénitas [Internet] 2016 [actualizado 7 septiembre de 2016; consultado 20 de diciembre de 2018]. Disponible en: http://www.who.int/es/news-room/factsheets/detail/congenital-anomalies

2. Navarrete E, Canún S, Valdés J, Reyes A. Malformaciones congénitas al nacimiento: México 2008-2013. Bol Med Hosp Infant Mex. [Internet] 2017 [consultado: 16 de diciembre de 2018];74(4): 301-8. Disponible en: https://doi.org/10.1016/j.bmhimx.2017.02.003

3. Gil M, Miguel M, Rosas M. Síndrome de obstrucción intestinal neonatal. Revista Mexicana de Atención Primaria a la Salud. [Internet] 2016 [consultado: 8 de enero de 2019];4:18-21. Disponible en: https://bit.ly/3gTKEJA

4. Miscia M, Lauriti G, Lelli P, Zani A. Duodenal atresia and associated intestinal atresia: a cohort study and review of the literature. Pediatr Surg Int. [Internet] 2018 [consultado: 29 de enero de 2019]; 35(1): 151-7. Disponible en: https://doi.org/10.1007/s00383-018-4387-1

5. Chiarenza S, Bucci V, Conighi M, Zolpi E, Costa L, Fasoli L, Bleve C. Duodenal atresia: open versus mis repair analysis of our experience over the last 12 years. BioMed Res Int. [Internet] 2017 [consultado: 29 de enero de 2019]; 1-7. Disponible: https://doi.org/10.1155/2017/4585360

6. Siu A, Paredes R, Betancourth J, Vázquez F, Delgado L, Garrido J. Análisis retrospectivo de la morbilidad y mortalidad de las atresias intestinales diagnosticadas en el periodo neonatal. Cir Pediatr. [Internet]. 2018 [consultado: 17 de enero de 2019]; 31(2): 85-9. Disponible en: https://bit.ly/2XY9Jfb

7. Ormaechea M, Berazategui R. Membrana duodenal: a propósito de un caso. AnFaMed, Universidad República de Uruguay. [Internet] 2017 [consultado: 20 de enero de 2019]; 4(2): 47-57. Disponible en: https://doi.org/10.25184/anfamed2017v4n2a7

8. Lara D. Intervenciones especializadas en las necesidades básicas de un neonato a término con atresia esofágica. [Tesis de especialidad]. México: Universidad Nacional Autónoma de México; 2017 [consultado: 19 de enero de 2019]. Disponible en: http://132.248.9.195/ptd2017/octubre/0766799/Index.html

9. Montero W. Estudio de caso: recién nacido con alteración en la necesidad de alimentación por atresia de esófago grado III. [Tesis de especialidad]. México: Universidad Nacional Autónoma de México; 2017 [consultado: 18 de enero de 2019]. Disponible en: http://132.248.9.195/ptd2017/junio/0760201/Index.html 
10. Mondragón I, Sosa M. Experiencias de los alumnos en el aprendizaje del proceso de atención de enfermería. Enferm Univ. [Internet] 2019 [consultado: 7 de febrero 2019]; 16(3): 249-58. Disponible en:

https://doi.org/10.22201/eneo.23958421e.2019.3.709

11. Gordon M. Diagnóstico enfermero. Proceso y Aplicación. 3ra ed. Madrid, España: Harcourt Brace; 1999.

12. Herdman TH, Kamitsuru S. NANDA Internacional, Inc. Diagnósticos enfermeros. Definiciones y clasificación 20182020. Edición hispanoamericana. 11 ed. España: Elsevier; 2019.

13. Moorhead S, Johnson M, Swazon E, Maas M. Clasificación de resultados de enfermería (NOC). 6a ed. Barcelona España: Elsevier-Mosby; 2018.

14. Butcher H, Bulechelk G, Docheterman J, Wagner C. Clasificación de Intervenciones de Enfermería (NIC). 7a ed. Barcelona España: Elsevier; 2018.

15. Secretaría de Salud (SS). Guía de práctica clínica manejo integral de heridas de alta complejidad en el paciente pediátrico en los tres niveles de atención a la salud (GPC SS-745-15). [Internet] 2015 [consultado: 17 de diciembre de 2018]. Disponible en: https://n9.cl/3u2d

16. Secretaría de Salud (SS). Guía de práctica clínica prevención, diagnóstico y tratamiento de sepsis y choque séptico del recién nacido en el segundo y tercer nivel de atención (SS-283-12). [Internet] 2012 [consultado: 16 de diciembre de 2018]. Disponible en: https://n9.cl/fwj7

17. Alfaro R. Pensamiento crítico y juicio clínico en enfermería. un enfoque práctico para un pensamiento centrado en los resultados. 4a. Ed. España: Elsevier; 2009.

Cómo citar este artículo: Sánchez-Juárez IA, De Avila-Arroyo ML, Tenahua-Quitl I, Torres-Reyes A. Proceso enfermero en neonato con retraso en la recuperación quirúrgica por atresia duodenal. SANUS [Internet]. 2021 [citado el $\underline{\mathrm{dd}} \underline{\mathrm{mm}}$ aa]; 6: e189. Disponible en: https://doi.org/10.36789/sanus.vi1.189 